\title{
Colonic bleeding due to rupture of an isolated iliac artery aneurysm into a caecal carcinoma
}

\author{
D. Aladgem, A. Mazor, H. Kashtan, N. Ostrzega, Y. Barak and T. Wiznitzer \\ Department of Surgery 'A', Ichilov Hospital, Tel-Aviv Medical Center, The Sackler School of Medicine, \\ Tel-Aviv 64239, Israel.
}

\begin{abstract}
Summary: We describe a case of a fistula between. an atherosclerotic aneurysm of the right common iliac artery and a caecal carcinoma, causing massive colonic bleeding. A fistula between a true aneurysm and a carcinoma of the colon has not to our knowledge been previously reported. Various aspects of this case are discussed from a pathogenetic point of view.
\end{abstract}

\section{Introduction}

Gastrointestinal bleeding from an aorto-enteric fistula can result from spontaneous rupture of an aneurysm into an adjacent viscus. This can be a late complication of surgical replacement, or bypass of the aorto-iliac vessels, ${ }^{1}$ and usually involves the duodenum. We report a rare case of atherosclerotic aneurysm which ruptured into a caecal carcinoma, with fatal outcome due to uncontrolled colonic bleeding.

\section{Case report}

A 78 year old male was admitted with rectal bleeding and syncope. At the age of 53 he underwent right orchidectomy for seminoma, followed by irradiation therapy. Five days before admission, he suffered rectal bleeding which continued until he fainted and was brought to hospital. Physical examination revealed a very pale, elderly male, with a pulse rate of $110 / \mathrm{min}$ and blood pressure of $90 /$ $40 \mathrm{~mm} \mathrm{Hg}$. Oedema was noticed in the right lower extremity, radiation dermatitis over the right lower abdominal quadrant and an irregular, immobile mass was palpable through the right lower abdominal wall. A rectal examination revealed fresh blood, and a non-pulsating mass was palpable through the right anterior rectal wall. Gastric aspirate showed bile stained gastric juice.

Soon after admission, he continued to experience massive rectal bleeding. Despite aggressive resuscitation with fluids and blood, the patient developed signs of cerebral damage and refractory shock, and died a few hours later following cardiorespiratory arrest.

Correspondence: H. Kashtan M.D.

Accepted: 29 February 1988
At autopsy, $3000 \mathrm{ml}$ of sanguinous fluid with blood clots were found in the peritoneal cavity, as well as in the colon. Multiple fibrous adhesions were present between small and large bowel, predominantly in the area of the caecum, where a mass adherent to the right common iliac artery was found. The mass, on dissection, was found to be an isolated fusiform aneurysmal dilatation of the right common iliac artery which had ruptured into the adherent caecum, forming a fistula, measuring $7 \mathrm{~mm}$ in diameter.

The fistula's tract communicated also with the abdominal cavity. There was no macroscopic evidence of a caecal tumour. A few diverticula were found in the transverse colon. There was severe atherosclerosis involving the abdominal aorta and both iliac arteries. Retroperitoneal fibrosis was present on the right side, with obstruction of the right ureter and corresponding upper urinary tract dilatation. Macro- and micronodular cirrhosis and hepatosplenomegaly were also found.

Microscopic examination confirmed the diagnosis of an atherosclerotic aneurysm of the right common iliac artery. Sections from the adherent caecal wall showed the presence of an anaplastic carcinoma infiltrating through the entire caecal wall and into the pericolic fat. The cancer appeared to originate in the colonic mucosa. There was no histological evidence of malignancy either in the iliac artery wall or in the fistula tract.

\section{Discussion}

In more than $80 \%$ of patients, the source of massive lower gastrointestinal bleeding is either pancolic diverticula or angiodysplasia of the caecum and ascending colon. Spontaneous atheroscler-

(C) The Fellowship of Postgraduate Medicine, 1988 
otic fistula, as a cause of massive gastrointestinal bleeding is relatively rare and usually fatal. The first reported case of an aortic aneurysm rupturing into the duodenum is usually credited to Salmon ${ }^{2}$ in 1843, although the condition was known to surgeons before that time. Tyrell, who published the lectures of Sir Astley Cooper in 1839, recorded a case presentation which he made to his students, in which he described a case of aortoenteric fistula. $^{3}$

Fistula formation may occur between an aortic aneurysm and all parts of the gastrointestinal tract. Reckless et al., ${ }^{1}$ in their review of 131 cases, found that fistulization to the duodenum was most frequent $(81.6 \%)$, followed by small bowel $(8 \%)$, large bowel $(5.6 \%)$ and the stomach $(4.8 \%)$. Aortic aneurysms can also rupture into the ureters ${ }^{4}$ and the appendix. ${ }^{5}$ Rupture into the gut from aneurysm in the arteries other than the aorta, is rare and only a few cases have been reported.4,6-10

Usually, an aortoenteric fistula develops following an operation to the abdominal aorta or its branches. This late complication occurs in about $1 \%\left(0.6-4 \%^{11,12}\right)$ of cases. Nowadays, primary and spontaneous aortoenteric fistulas are even rarer because of early elective resection of abdominal aneurysms.

Our case had a combination of unusual features. The rate of the bleeding was too fast to enable resuscitation, diagnostic procedures and treatment. In most cases of arterio-intestinal fistulas, the bleeding usually starts slowly enabling a diagnostic workup and planning of surgical treatment. ${ }^{4,13}$ Our

\section{References}

1. Reckless, J.P.D., McColl, I. \& Taylor, G.W. Aortoenteric fistula an uncommon complication of abdominal aortic aneurysm. Br J Surg 1972, 59: 458-460.

2. Salmon, A. Aneurysme de l'aorte ventral: mort par

- rupture de poche arterielle dans le duodenum. Bull Soc Anatomic de Paris 1943, 18: 283.

3. Cooper, A. The lectures of Sir Astley Cooper on the principles and practice of surgery. with additional notes and cases by F. Tyrrel, 5th American Ed. Haswell. Barrington and Haswell, Philadelphia, 1839.

4. Macbeth, B.A. Rupture of abdominal aneurysms into the gastrointestinal tract. Can J Surg 1962, 5: 372-378.

5. Bisberg, B. \& Kewerter, J. Arterio-appendiceal fistula after arterial reconstruction with synthetic graft. Acta Chir Scand 1978, 144: 121-123.

6. Atin, H.L. Rupture of an iliac artery aneurysm into sigmoid colon. Report of a case. N Engl J Med 1958, 258: 366.

7. Jackman, R.J., McQuarric, H.B. \& Edwards J.E. Fatal rectal hemorrhage caused by an aneurysm of the internal iliac artery. Report of a case. Mayo Clin Proc 1948, 23, 305-308. patient was bleeding for 5 days before he was referred to us.

The circumstances usually described involve a pseudoaneurysm invading the healthy bowel ${ }^{14}$ and the mechanism suggested is that when bowel remains attached to an aortic graft or suture line, it is subjected to repeated trauma with each aortic pulsation. If this trauma continues, the bowel wall may eventually erode; the subsequent spillage of intestinal contents leads to infection in the graft, and breakdown of the anastomosis itself may follow. Even when there is no infection, a false aneurysm may form at a tenuous vascular suture line. This aneurysm may then involve the neighbouring bowel and lead to fistula formation. Another possibility is that a true pre-existing aneurysm may adhere to an inflammatory process in the intestine and be further complicated by fistula formation. 9,15

The combination of gastrointestinal fistula consisting of a true aneurysm and carcinoma of the colon has, to the best of our knowledge, never been described before.

Delayed effect of radiotherapy possibly played a role in our case. The combination of radiation dermatitis, femoral vein thrombosis, right retroperitoneal fibrosis with hydroureter and hydronephrosis, and carcinoma of the caecum, all in the same irradiated area, strongly indicates radiation injury. The possibility that this injury played a role in both the development and rupture of the aneurysm into the adherent caecal carcinoma, should also be considered.

8. Howard, C. \& Baron, M.D. Isolated aneurysm of internal iliac artery producing rectal bleeding. $N Y$ State J Med 1979, Nov: 79: 1884-1886.

9. Longakers, C.J., Bubrick, M.P. \& Kiser, J.C. Arteriocolic fistula, an unusual case of gastrointestinal tract bleeding. Dis Colon Rectum 1977, 20: 135-138.

10. Smeesters, C.J. \& Queneville, G. An unusual case of primary arteriocolic fistula. Dis Colon Rectum 1981, 24: 305-307.

11. Elliot, J.P., Smith, R.F. \& Scilaggi, D.E. Aortoenteric and pan-prosthetic enteric fistulae: problems of diagnosis and management. Arch Surg 1974, 108, 479-490.

12. Levy, M.J., Todd, T.B., Lillehi, C.W. \& Varco, R.L. Aortointestinal fistula following surgery of the aorta. Surg Gynecol Obstet 1965, 120: 992-996

13. Voylas, W.R. \& Maretz, W.H. Rupture of aortic aneurysms into gastrointestinal tract. Surgery 1958, 43: 666 .

14. Baird, R.L. \& Slagle, G.W. Arteriosclerotic fistulas Dis Colon Rectum 1979, 22: 187-188.

15. Wilson, S.E. \& Owens, M.L. Aortocolic fistula, a lethal cause of lower gastrointestinal bleeding. Dis Colon Rectum 1976, 19: 614-617. 